\title{
Superoxide Dismutase Detection in Human Cumulus Oophorus Cells
}

L. Matos, ${ }^{* * *}$ D. Stevenson, ${ }^{* * *}$ F. Gomes, ${ }^{* * *}$ J. L. Silva-Carvalho, ${ }^{* * *}$ H. Almeida ${ }^{*}$

* Laboratório de Biologia Celular e Molecular, Faculdade de Medicina, Alameda Prof. Hernâni Monteiro, 4200-319 Porto, and IBMC - Instituto de Biologia Molecular e Celular, Universidade do Porto, Rua do Campo Alegre 823, 4150-180 Porto, Portugal

** Faculdade de Ciências da Nutrição e Alimentação, Universidade do Porto, Rua Dr. Roberto Frias, 4200-465 Porto, Portugal

*** Unit of Reproductive Medicine, Department of Gynaecology and Obstetrics, Faculdade de Medicina do Porto/ Hospital S. João, Alameda Prof. Hernâni Monteiro, 4200-319 Porto, Portugal almeidah@med.up.pt

A key factor limiting the success of Assisted Reproduction Techniques (ART) is oocyte and embryo quality, usually assessed by their morphologic appearance. Due to the subjectivity and inaccuracy of such criteria, other predictors of oocyte and embryo quality would be welcome. Cumulus oophorus (c.o.) cells are eligible as oocyte quality predictors, due to their direct contact with it. In addition, ART success was also related to oxidative stress, whose effects in the porcine and bovine oocytes may be prevented by the presence of c.o. cells [1]. Yet, it is unknown if a similar effect exists in humans due to the scarcity of studies employing human c.o. cells.

In the present study, human c.o. cells discarded from common ART were processed for their morphological characterization and to assess antioxidants as Superoxide Dismutase (SOD). Culture conditions were similar to those used for human oocyte in vitro maturation studies [2], optimized for a better achievement of cell proliferation. Cell structure was assessed at light and electron microscopy level, using current processing protocols. Some samples of cells were studied by immunocytochemistry to detect the mitochondrial manganese isoform of SOD (MnSOD).

Cells in vitro rapidly adhere, forming cytoplasmic processes before proliferating. Initially, they were round and small but later acquired a fibroblast-like morphology, exhibiting larger dimensions and long extensions. Their lipid droplet and endoplasmic reticulum content suggest a steroidogenic ability. On day 5, the droplets were bigger and more abundant (Fig. 1A, 1C) but on day 15 they become smaller and lesser (Fig. 1B, 1D), possibly as a result of steroid synthesis. MnSOD was found in all cells, showing a granular stippling cytoplasmic pattern, agreeing with its mitochondrial localization (Fig. 2). The ultrastructural study confirmed the structural findings (Fig. 3).

As conclusion, human c.o. cells may be cultured in vitro and processed for morphological study. MnSOD immunocytochemistry staining pattern agrees with its mitochondrial localization. Growing c.o. cells in culture may allow the evaluation of additional markers that might improve oocyte and embryo quality assessment and ART success.

\section{References}

[1] A. N. Fatehi et al., Zygote, 13 (2005) 157.

[2] C. M. H. Combelles et al., Human Reproduction, 20 (5) (2005) 1349.

[3] This work was supported by a grant from Sociedade Portuguesa de Ginecologia. 

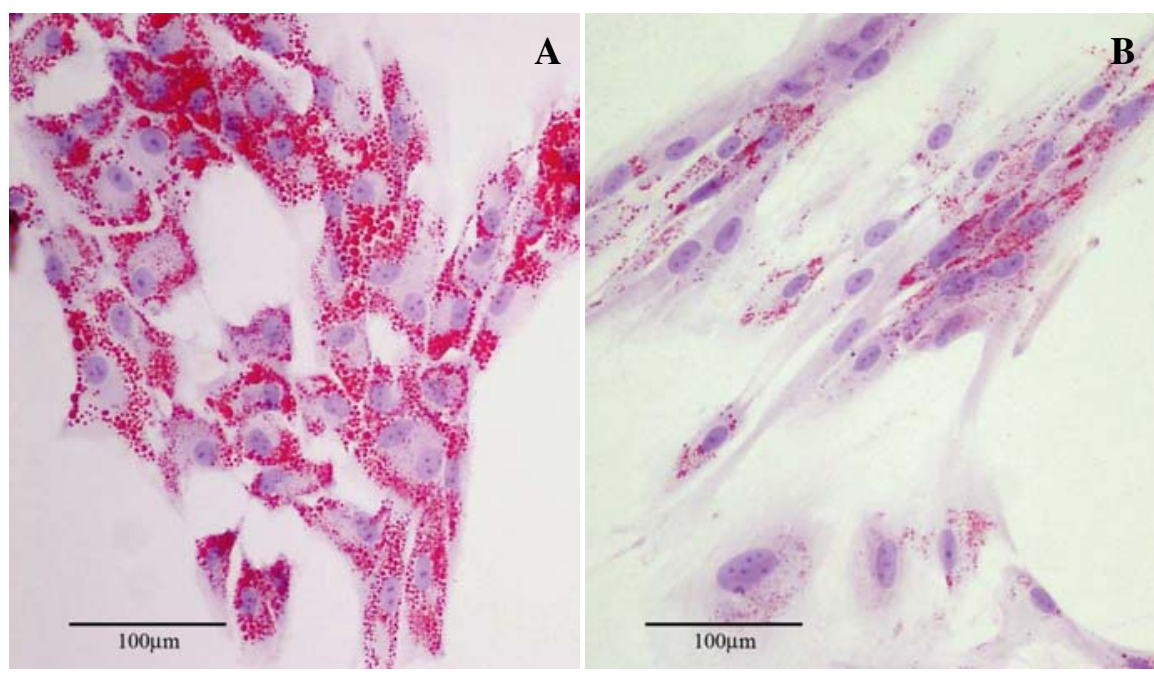

Fig. 1. Cumulus Oophorus cells in culture stained for lipid droplets with Sudan III/IV on day 5 (A and C) and 15 (B and D).
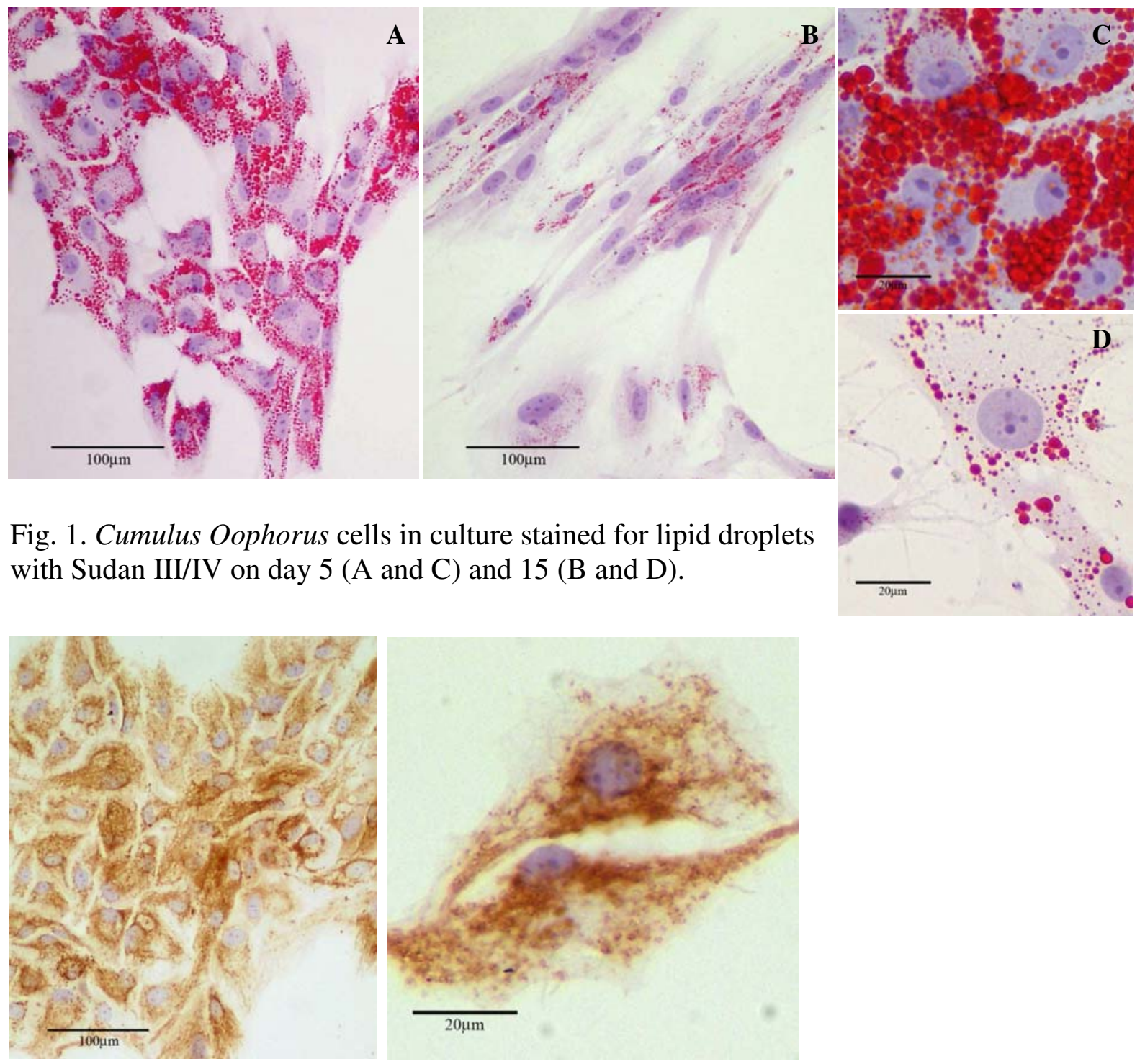

Fig. 2. MnSOD immunolocalization in cultured cumulus oophorus cells.
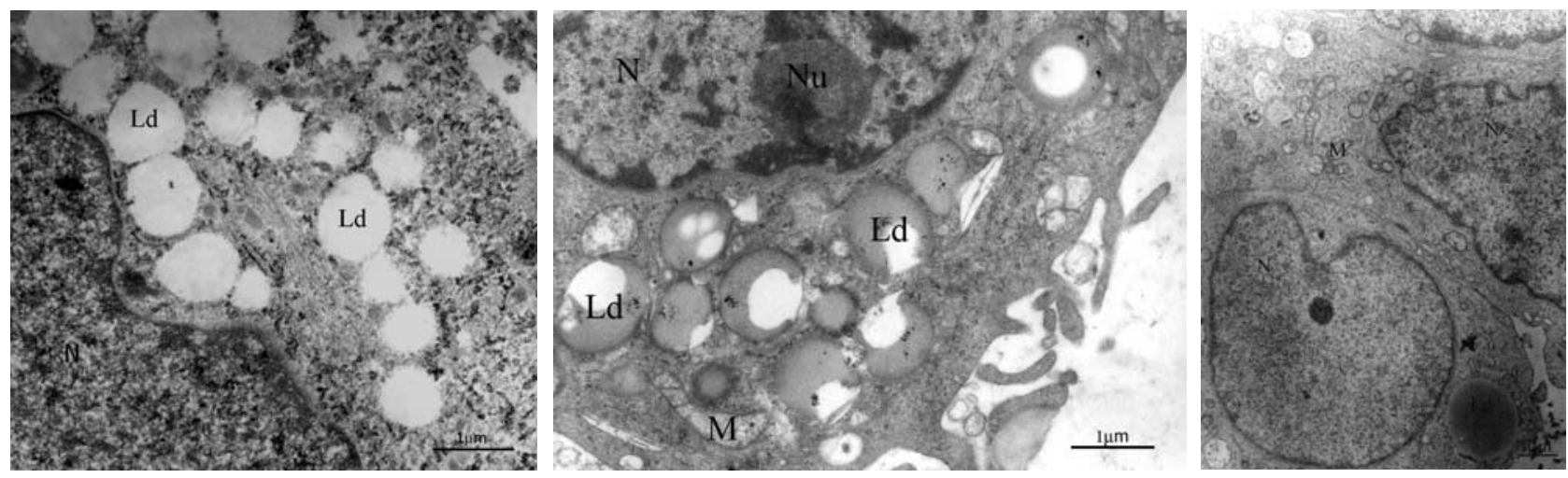

Fig. 3. Cumulus oophorus ultrastructure. Ld - Lipid droplet; M- mitochondria; N- Nucleus; NuNucleolus. 